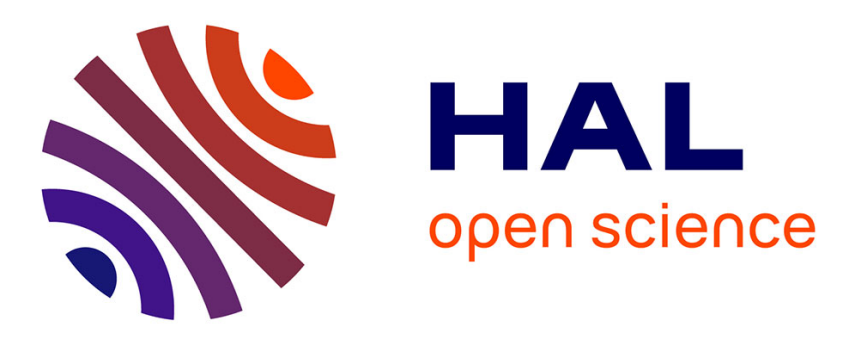

\title{
The MID property for a second-order neutral time-delay differential equation
}

\author{
Amina Benarab, Islam Boussaada, Catherine Bonnet, Karim Trabelsi, \\ Guilherme Mazanti
}

\section{- To cite this version:}

Amina Benarab, Islam Boussaada, Catherine Bonnet, Karim Trabelsi, Guilherme Mazanti. The MID property for a second-order neutral time-delay differential equation. 24th International Conference on System Theory, Control and Computing, Oct 2020, Sinaia, Romania. 10.1109/ICSTCC50638.2020.9259685 . hal-02934532

\section{HAL Id: hal-02934532 \\ https://hal.science/hal-02934532}

Submitted on 9 Sep 2020

HAL is a multi-disciplinary open access archive for the deposit and dissemination of scientific research documents, whether they are published or not. The documents may come from teaching and research institutions in France or abroad, or from public or private research centers.
L'archive ouverte pluridisciplinaire HAL, est destinée au dépôt et à la diffusion de documents scientifiques de niveau recherche, publiés ou non, émanant des établissements d'enseignement et de recherche français ou étrangers, des laboratoires publics ou privés. 


\section{The MID property for a second-order neutral time-delay differential equation}

\author{
Amina Benarab \\ IPSA \& Université Paris-Saclay, L2S \\ CNRS-CentraleSupélec, Inria \\ Gif-sur-Yvette, France \\ amina.benarab@ipsa.fr
}

Guilherme Mazanti

Université Paris-Saclay, L2S

CNRS-CentraleSupélec, Inria

Gif-sur-Yvette, France

guilherme.mazanti@inria.fr

\author{
Islam Boussaada \\ Université Paris-Saclay, L2S \\ CNRS-CentraleSupélec, Inria \\ Gif-sur-Yvette, France \\ islam.boussaada@inria.fr
}

\author{
Catherine Bonnet \\ Université Paris-Saclay, L2S \\ CNRS-CentraleSupélec, Inria \\ Gif-sur-Yvette, France \\ catherine.bonnet@inria.fr
}

\begin{abstract}
This paper considers the Multiplicity-Induced-Dominancy (MID) property for second order neutral time-delay differential equations. Necessary and sufficient conditions for the existence of a root of maximal multiplicity are given in terms of this root and the parameters (including the delay) of the given equation. Links with dominancy of this root and with the exponential stability property of the solution of the considered equations are given. Finally, we illustrate the obtained results on the classical oscillator control problem.
\end{abstract}

Index Terms - neutral delay system, dominant root, root of maximal multiplicity, exponential stability

\section{INTRODUCTION}

Systems with time delays provide useful models in a wide range of scientific and technological domains such as biology, chemistry, economics, physics, or engineering, where the presence of the delays is inherent to propagation phenomena, such as of material, energy, or information, with a finite propagation speed. Due to their numerous applications, these kinds of systems have been the subject of much attention by researchers in several fields, in particular since the 1950s and 1960s. We refer to [15], [16], 24] for details on time-delay systems and their applications.

Linear systems with delays are described in the Laplace domain by transfer functions involving quasipolynomials and then admit an infinite number of poles. Studying the stability properties of retarded

CB and IB are partially supported by a public grant overseen by the French National Research Agency (ANR) as part of the "Investissement d'Avenir" program, through the iCODE project funded by the IDEX Paris-Saclay, ANR-11-IDEX000302. systems (they admit a finite number of poles in any right half-plane) is much easier than studying those of neutral systems which may have an infinite number of poles, in chains asymptotic to a vertical axis possibly located in the open right half-plane or clustering the imaginary axis from left or right. Both situations prevent to get exponential stability for these systems.

To perform stability analysis, efficient methods have been proposed in frequency-domain, see, for instance, [1], [11], 16], 25], 29]-31 and the references therein. Even with the significant advances that have been reported on such topics, the question of determining conditions on the equation parameters that guarantee asymptotic stability of solutions of linear time-invariant time-delay systems remains still open.

Once stability conditions are established, further questions related to performance occur. For instance, what is the location of the corresponding rightmost root 1 of the system characteristic equation?

The starting point of the present work is a property, discussed in recent studies, called MultiplicityInduced-Dominancy, see for instance [6]. As a matter of fact, it is shown that multiple spectral values for time-delay systems can be characterized using a Birkhoff/Vandermonde-based approach; see for instance [2] [4], 10]. More precisely, in previous works, it is emphasized that the admissible multiplicity of the real spectral values is bounded by the generic Polya and Szegö bound (denoted $P S_{B}$ ), which is nothing but the degree of the corresponding quasipolynomial (i.e the sum of the degrees of the involved

\footnotetext{
${ }^{1}$ Such a rightmost root corresponds to the so-called $\alpha$ stability problem, itself related to the solution's decay rate.
} 
polynomials plus the number of delays), see for instance [27, Problem 206.2, page 144 and page 347]. It is worth mentioning that such a bound was recovered using structured matrices in $[3]$ rather than the argument principle as in 27. It is important to point out that the multiplicity of a root itself is not relevant as such, however its connection with the eventual induced dominancy is a meaningful tool for control synthesis. To the best of the authors' knowledge, the first time an analytical proof of the dominancy of a spectral value for the scalar equation with a single delay was presented and discussed in the $50 \mathrm{~s}$, see 17. The dominancy property is further explored and analytically shown in scalar delay equations in [10], then in second-order systems controlled by a delayed proportional controller in 7], 9], where its applicability in damping active vibrations for a piezoactuated beam is proved. An extension to the delayed proportional-derivative controller case is studied in [5], [8] where the dominancy property is parametrically characterized and proven using the argument principle. See also [5], 8] which exhibit an analytical proof for the dominancy of the spectral value with maximal multiplicity for second-order systems controlled via a delayed proportional-derivative controller.

Lately, the MID property has been extended to neutral differential equations, first in 21] in the context of the PID controller design for first-order delayed-plants, then in 23] where the MID occurs for spectral values with maximal multiplicity in generic scalar neutral differential equations.

The paper is organized as follows: Section [II provides some motivation examples as well as the problem formulation. Section [II exhibits some technical results on the stability and large-time behaviour of neutral equations. Section IV states the main results of this study. Finally, Section $\mathrm{V}$ is dedicated to the exponential stabilization of an oscillator via delay, as an illustrative example.

\section{Motivations and Problem Setting}

This section is dedicated to recalling some recent results on the MID property for first and second-order neutral differential equations.

A. MID property for first-order scalar neutral equations

It is shown in 23 that the MID property holds for the delay differential algebraic system

$$
\left\{\begin{array}{l}
\dot{x}(t)=a x(t)+b y(t-\tau), \\
y(t)=c x(t)+d y(t-\tau),
\end{array}\right.
$$

where $x(t)$ and $y(t)$ are real-valued, and $a, b, c, d$ are real coefficients, and whose characteristic function is given by

$$
\Delta(s)=s-a-\mathrm{e}^{-s \tau}(s d-a d+b c) .
$$

As a matter of fact, the maximal multiplicity, which is equal to 3 , is reached at $s_{0} \in \mathbb{R}$, and expressions of the coefficients ensuring such a configuration are determined in terms of $s_{0}$ and the delay $\tau$. Furthermore, all complex roots of (2) with real-parts equal to $s_{0}$ are fully characterized.

\section{B. Applicative interest in MID property: Robust PID stabilizing design for first-order delayed plants}

The work in 19 aims at extending such a design approach to time-delay systems of neutral type occurring in the classical problem of PID stabilizing design for delayed plants. Namely, consider the following closed-loop plant

$$
M(s)=\frac{\left(k_{d} s^{2}+k_{p} s+k_{i}\right) \mathrm{e}^{-s \tau}}{s^{2}-p s+\left(k_{d} s^{2}+k_{p} s+k_{i}\right) \mathrm{e}^{-s \tau}},
$$

where $p$ is a positive unstable pole of the open-loop plant, $k_{p}, k_{i}, k_{d}$ are real parameters (gains) and $\tau$ is the delay. In 28], it was found that the delay margin is $\tau_{P I D}=\frac{2}{p}$; see also 20 . Now, the corresponding characteristic function is given by

$$
\Delta(s)=s^{2}-p s+\mathrm{e}^{-s \tau}\left(k_{d} s^{2}+k_{p} s+k_{i}\right) .
$$

In [19], it is shown that for arbitrary real parameters $k_{p}, k_{i}, k_{d}$ and arbitrary positive delay $\tau$, the multiplicity of a given root of (4) is bounded by 4 . In addition, the maximal multiplicity 4 is only reached by two roots $s_{ \pm}$for one set of given values of the gains. As a result, if $\tau<\tau_{P I D}$, then the root $s_{+}$is dominant and guarantees stability.

\section{Does the maximal multiplicity guarantee the domi-} nancy for second-order neutral differential equations?

A natural question arises. Can one extend the result of Mazanti et al [23] to second-order neutral differential equations. Hence, consider the generic second-order neutral delay differential equation with a single delay,

$$
\left\{\begin{array}{l}
\sum_{k=0}^{2} a_{k} \frac{\mathrm{d}^{k} x}{\mathrm{~d} t^{k}}(t)+\alpha_{k} \frac{\mathrm{d}^{k} x}{\mathrm{~d} t^{k}}(t-\tau)=0, \\
x(t)=\varphi(t), \quad-\tau \leq t \leq 0,
\end{array}\right.
$$

where $a_{2}=1, a_{0}, a_{1}, \alpha_{0}, \alpha_{1}, \alpha_{2} \in \mathbb{R}, \alpha_{2} \neq 0, \tau>0$, and $\varphi$ is a given continuously differentiable realvalued history function on the initial interval $[-\tau, 0]$. Its characteristic function is given by the following quasipolynomial of degree 5 ,

$$
\Delta(s)=s^{2}+a_{1} s+a_{0}+\left(\alpha_{2} s^{2}+\alpha_{1} s+\alpha_{0}\right) \mathrm{e}^{-\tau s} .
$$


In other words, we shall investigate the validity of the multiplicity-induced-dominancy (MID) property for the above class of quasipolynomial functions.

\section{Prerequisites}

In this section, we recall the basic spectral theory for linear functional differential equations. Let $\mathcal{C}=$ $\mathcal{C}\left([-\tau, 0], \mathbb{C}^{n}\right)$ denote the Banach space of continuous functions endowed with the supremum norm. For a function $X:[-\tau, \infty) \rightarrow \mathbb{C}^{n}$, we denote by $X_{t} \in \mathcal{C}$ the function $X_{t}(\theta)=X(t+\theta),-\tau \leq \theta \leq 0$ and $t \geq 0$.

An initial value problem for a linear autonomous neutral functional differential equation is given by the following relation

$$
\begin{cases}\frac{\mathrm{d}}{\mathrm{d} t} D X_{t}=L X_{t}, & t \geq 0 \\ X_{0}=\phi, & \phi \in \mathcal{C}\end{cases}
$$

where $\phi$ is the prior data, $D: \mathcal{C} \rightarrow \mathbb{C}^{n}$ is continuous, linear and atomic at zero, $L: \mathcal{C} \rightarrow \mathbb{C}^{n}$ is linear and continuous and both operators are, owing to the Riesz representation theorem, defined by

$L \phi=\int_{-\tau}^{0} \mathrm{~d} \eta(\theta) \phi(\theta)$ and $D \phi=\phi(0)-\int_{-\tau}^{0} \mathrm{~d} \mu(\theta) \phi(\theta)$,

where $\mu, \eta \in N B V\left([-\tau, 0], \mathbb{C}^{n \times n}\right)$ are $\mathbb{C}^{n \times n}$ matrices the elements of which are of bounded variation, normalized so that $\mu$ is continuous at zero and $\eta(0)=0$; see Hale and Verduyn Lunel [16] for details.

Remark 1. Note that it suffices to let $y(t)=x^{\prime}(t)$ in (5), and set $X_{t}={ }^{T}(x(t) y(t))$ and $\phi={ }^{T}\left(\varphi \varphi^{\prime}\right)$ to reframe our problem as above:

$$
X^{\prime}(t)-B X^{\prime}(t-\tau)=-A_{0} X(t)+A_{1} X(t-\tau),
$$

where

$$
\begin{gathered}
B=\left(\begin{array}{cc}
0 & 0 \\
0 & -\alpha_{2}
\end{array}\right), A_{0}=\left(\begin{array}{cc}
0 & -1 \\
a_{0} & a_{1}
\end{array}\right), \\
A_{1}=\left(\begin{array}{cc}
0 & 0 \\
-\alpha_{0} & -\alpha_{1}
\end{array}\right) .
\end{gathered}
$$

More precisely, as we are dealing with one discrete delay $\tau>0$ in our case, one has

$\mu(\theta)=\left\{\begin{array}{cl}-B, & \theta \leq-\tau, \\ 0, & \theta>\tau,\end{array} \quad \eta(\theta)=\left\{\begin{array}{cl}-A_{1}, & \theta \leq-\tau, \\ 0, & -\tau<\theta<0, \\ -A_{0}, & \theta \geq 0\end{array}\right.\right.$

It is well-known that for any given initial function $\phi$, there exists a unique solution of the initial value problem (7); see [12]. Namely, given the solution $X(\phi)$ of the initial value problem (7), we define the solution operator $T(t): \mathcal{C} \rightarrow \mathcal{C}$ by the relation

$$
T(t) \phi=X_{t}(. ; \phi), \quad t \geq 0 .
$$

Hale and Verduyn Lunel [16] proved that the solution operator is a $\mathrm{C}_{0}$-semigroup on $\mathcal{C}$, its infinitesimal generator $A$ being

$$
\left\{\begin{array}{l}
D(A)=\left\{\phi \in \mathcal{C} \mid \frac{\mathrm{d} \phi}{\mathrm{d} \theta} \in \mathcal{C}, D \frac{\mathrm{d} \phi}{\mathrm{d} \theta}=L \phi\right\} \\
A \phi=\frac{\mathrm{d} \phi}{\mathrm{d} \theta} .
\end{array}\right.
$$

Moreover, $\sigma(A)=P_{\sigma(A)}$ and $s \in \sigma(A)$ if, and only if, $s$ satisfies the characteristic equation $\operatorname{det} \mathbb{M}(s)=0$, $\mathbb{M}$ being the characteristic matrix

$$
\mathbb{M}(s)=s I-\int_{-\tau}^{0} s \mathrm{e}^{s \theta} \mathrm{d} \mu(\theta)-\int_{-\tau}^{0} \mathrm{e}^{s \theta} \mathrm{d} \eta(\theta),
$$

and $P_{\sigma(A)}$ the point spectrum of $A$.

\section{A. Asymptotic behavior by the spectral approach}

For a given $s$ in the spectrum of $A$, let define $\mathcal{M}_{s}$ by the generalized eigenspace associated to $s$ :

$$
\mathcal{M}_{s}=\mathcal{N}(s I-A)^{\kappa_{s}},
$$

where $\kappa_{s}$ is the order of $z=s$ as a pole of $\mathbb{M}^{-1}(z)$. From the spectral theory [16], it follows that the spectral projection onto $\mathcal{M}_{s}(A)$ along $\mathcal{R}\left((s I-A)^{\kappa_{s}}\right)$ can be represented by a Dunford integral

$$
P_{s}=\frac{1}{2 i \pi} \int_{\Gamma_{s}}(z I-A)^{-1} \mathrm{~d} z,
$$

where $\Gamma_{s}$ is a small circle such that $s$ is the only singularity of $(z I-A)^{-1}$ inside. Finally, Frasson 13 shows that if $s_{0} \in \mathbb{R}$ is a dominant zero of $\mathbb{M}(s)$ of multiplicity $n \geq 1$, then

$$
P_{s_{0}} \phi=0 \Longrightarrow \lim _{t \rightarrow \infty} \mathrm{e}^{-t s_{0}} X(t)=0 .
$$

B. Insights on spectrum distribution for neutral delay systems

The generic form of the transfer function of a neutral delay system is

$$
G(s)=\frac{r(s)}{p(s)+q(s) \mathrm{e}^{-s \tau}},
$$

where $p, q$ and $r$ are real polynomials such that $\operatorname{deg} p=\operatorname{deg} q$ and $\tau>0$. Let $\alpha=\lim _{|s| \rightarrow \infty} \frac{p(s)}{q(s)}$ assumed to be a nonzero real number. The case $|\alpha| \neq 1$ is easily disposed of, as follows.

Proposition 1 ( 26$])$. For all $\tau>0$, the following holds

1) If $|\alpha|<1$, then $G$ has infinitely many unstable poles, asymptotic to a vertical line $\Re(s) \approx$ $-\frac{1}{\tau} \log |\alpha|$ in the right half-plane;

2) If $|\alpha|>1$, then the poles of $G$ of large modulus are asymptotic to a vertical line strictly in the left half-plane; so that $G$ has at most a finite number of unstable poles. 


\section{Technical lemmas}

We conclude this section, by stating and proving technical results useful in the proof of the main result.

Following [23, Lemma 4.1.], the following lemma provides relations between the coefficients of $\Delta$ and those of the quasipolynomial $\hat{\Delta}$ obtained after the change of variable.

Lemma 1. Let $s_{0} \in \mathbb{R}$, and consider the quasipolynomial $\tilde{\Delta}: \mathbb{C} \rightarrow \mathbb{C}$ obtained from $\Delta$ by the following change of variables

$$
\tilde{\Delta}(z)=\tau^{2} \Delta\left(\frac{z}{\tau}+s_{0}\right), \quad z \in \mathbb{C} .
$$

Then

$$
\tilde{\Delta}(z)=z^{2}+M_{1} z+M_{0}+\left(N_{2} z^{2}+N_{1} z+N_{0}\right) \mathrm{e}^{-z},
$$

where

$$
\left\{\begin{array}{l}
M_{1}=\tau\left(2 s_{0}+a_{1}\right), M_{0}=\tau^{2}\left(s_{0}^{2}+a_{1} s_{0}+a_{0}\right), \\
N_{2}=\alpha_{2} \mathrm{e}^{-\tau s_{0}}, N_{1}=\tau\left(2 \alpha_{2} s_{0}+\alpha_{1}\right) \mathrm{e}^{-\tau s_{0}}, \\
N_{0}=\tau^{2}\left(\alpha_{2} s_{0}^{2}+\alpha_{1} s_{0}+\alpha_{0}\right) \mathrm{e}^{-\tau s_{0}}
\end{array}\right.
$$

Consider the following quasipolynomial function

$$
\hat{\Delta}(z)=z^{2}-6 z+12-\left(z^{2}+6 z+12\right) \mathrm{e}^{-z} .
$$

Following 22, Lemma 9], one obtains the following identity whose proof is straightforward.

Lemma 2. Let $\hat{\Delta}$ be given by (14). Then, one has

$$
\hat{\Delta}(-z)=-\mathrm{e}^{z} \hat{\Delta}(z), \quad z \in \mathbb{C} .
$$

An immediate consequence of the above identity is the following symmetry property for the roots of $\hat{\Delta}$.

Corollary 1. Let $\hat{\Delta}$ be given by (14) and assume that it has a root $z_{0} \in \mathbb{C}$. Then the following equalities hold

$$
\hat{\Delta}\left(z_{0}\right)=\hat{\Delta}\left(-z_{0}\right)=\hat{\Delta}\left(\bar{z}_{0}\right)=\hat{\Delta}\left(-\bar{z}_{0}\right)=0 .
$$

We conclude this section with the main technical ingredient.

Lemma 3. Let $\hat{\Delta}$ be given by (14) and assume that it has a root $z_{0} \in \mathbb{R}^{*}+i \mathbb{R}$. Then, $0<\Im\left(z_{0}\right)<\pi$.

\section{Main Results}

The main result we prove in this paper is the following.

Theorem 1. Consider the quasipolynomial

$$
\Delta(s)=s^{2}+a_{1} s+a_{0}+\left(\alpha_{2} s^{2}+\alpha_{1} s+\alpha_{0}\right) \mathrm{e}^{-\tau s} .
$$

1) The real $s_{0}$ is a root of multiplicity 5 of $\Delta$ if, and only if, the coefficients $a_{0}, a_{1}, \alpha_{0}, \alpha_{1}, \alpha_{2}$, the root $s_{0}$ and the delay $\tau$ satisfy the relations

$$
\left\{\begin{array}{l}
a_{1}=-2 s_{0}-\frac{6}{\tau}, a_{0}=s_{0}^{2}+\frac{6}{\tau} s_{0}+\frac{12}{\tau^{2}} \\
\alpha_{2}=-\mathrm{e}^{\tau s_{0}}, \alpha_{1}=\left(2 s_{0}-\frac{6}{\tau}\right) \mathrm{e}^{\tau s_{0}} \\
\alpha_{0}=-\left(s_{0}^{2}-\frac{6}{\tau} s_{0}+\frac{12}{\tau^{2}}\right) \mathrm{e}^{\tau s_{0}}
\end{array}\right.
$$

2) If 16 is satisfied, then $s_{0}$ is a dominant root of $\Delta$. Moreover, for all $s \in \mathbb{C}$, one has

$$
\Delta(s)=0 \quad \Longrightarrow \quad \Re(s)=s_{0} .
$$

3) If 16 is satisfied and $s_{0}<0$, then the trivial solution of (5) is asymptotically stable. In addition, if the history function $\phi={ }^{T}\left(\varphi \varphi^{\prime}\right)$ is chosen in order for its spectral projection with respect to the generalized $s_{0}$-eigenspace to vanish identically $P_{s_{0}} \phi=0$, then the large-time behaviour of the trivial solution of (5) is $\lim _{t \rightarrow \infty} \mathrm{e}^{-s_{0} t} x(t)=0$.

Remark 2. Note that item 3 of the theorem is obtained as a corollary of the MID property, unlike (12) in Frasson [13] where dominancy is assumed.

Remark 3. Since the expressions of $a_{0}, a_{1}, \alpha_{0}, \alpha_{1}$ and $\alpha_{2}$ in (16) are singular with respect to $\tau$ as $\tau \rightarrow 0$, should one be interested in studying the behavior of the roots of $\Delta$ as $\tau \rightarrow 0$ when (16) is satisfied, the quasipolynomial $\tau^{2} \Delta$ may be considered instead as it exhibits the same roots as $\Delta$, albeit with regular coefficients.

Before proceeding with the proof of the above theorem, it is convenient to normalize the setting using the affine change of variable $z=\tau\left(s-s_{0}\right)$. Consequently, the desired multiple root and the delay reduce to

$$
s_{0}=0 \quad \text { and } \quad \tau=1 \text {. }
$$

Remark 4. Note that under (17), relations (16) reduce to $a_{0}=12, a_{1}=-6, \alpha_{0}=-12, \alpha_{1}=$ $-6, \quad \alpha_{2}=-1$, so that the quasipolynomial (15) reduces to 14 .

Now, let us proceed with the proof of the main result.

Proof. Consider $\tilde{\Delta}$, the normalized quasipolynomial, it follows immediately from relation (14) that $s_{0}$ is a root of multiplicity 5 of $\Delta$ if, and only if, 0 is a root of multiplicity 5 of $\tilde{\Delta}$. As a matter of fact, since $\tilde{\Delta}$ is a quasipolynomial of degree 5 , zero is a root of multiplicity 5 of $\tilde{\Delta}$ if, and only if, $\tilde{\Delta}(0)=$ 
$\tilde{\Delta}^{\prime}(0)=\tilde{\Delta}^{(2)}(0)=\tilde{\Delta}^{(3)}(0)=\tilde{\Delta}^{(4)}(0)=0$. The latter identities yield the following Cramer system

$$
\left\{\begin{array}{l}
M_{0}+N_{0}=0, \\
M_{1}+N_{1}-N_{0}=0, \\
2+2 N_{2}-2 N_{1}+N_{0}=0, \\
-6 N_{2}+3 N_{1}-N_{0}=0, \\
12 N_{2}-4 N_{1}+N_{0}=0,
\end{array}\right.
$$

whose unique solution is $\left(M_{0}, M_{1}, N_{0}, N_{1}, N_{2}\right)=$ $(12,-6,-12,-6,-1)$ as required by (14), thereby ending the proof of the first item of the theorem. Moreover, note that, under (17), one has $\hat{\Delta}=\tilde{\Delta}$.

To prove the second item, it suffices to show that every root of $\hat{\Delta}$ lies on the imaginary axis. To do so, first, integration by parts yields

$$
\hat{\Delta}(z)=\frac{1}{2} z^{5} \int_{0}^{1} t^{2}(t-1)^{2} \mathrm{e}^{-z t} \mathrm{~d} t,
$$

then assume that there exists a root $z_{0} \in \mathbb{C}$ of $\hat{\Delta}$ satisfying $\Re\left(z_{0}\right) \neq 0$ and set to obtain a contradiction. Writing $z_{0}=\sigma+i \omega$ for $\sigma, \omega \in \mathbb{R}$ with $\sigma \neq 0$, one may assume, thanks to Corollary 1 below, that $\sigma>0$ and $\omega>0$. Next, using the fact that $z_{0}$ is a non-zero root of $\hat{\Delta}$, one infers from 18 , by taking the imaginary part, the identity below

$$
\int_{0}^{1} t^{2}(t-1)^{2} \exp (-\sigma t) \sin (\omega t) \mathrm{d} t=0 .
$$

Since $0<\omega \leq \pi$ by Lemma 3 , the function $t \mapsto$ $t^{2}(t-1)^{2} \exp -\sigma t \sin (\omega t)$ is strictly positive in $(0,1)$, which contradicts the above equality as required to end the proof.

The third item of the main result is a direct consequence of item two and 12); see Frasson [13.

\section{Illustrative Example: Exponential Stabilization OF AN OSCILLATOR USING DELAY ACTION}

Consider the classical oscillator control problem:

$$
\ddot{x}(t)+2 \xi \omega \dot{x}(t)+\omega^{2} x(t)=u(t)
$$

with $u$ as the delayed output-feedback as proposed in 18]: $u(t)=\alpha_{2} \ddot{x}(t-\tau)+\alpha_{1} \dot{x}(t-\tau)+\alpha_{0} x(t-\tau), \omega$ is the frequency of the arising vibrations and $\xi$ is the damping factor.

We proceed as in Remark 1 to reframe the problem as (8) with

$$
B=\left(\begin{array}{cc}
0 & 0 \\
0 & \alpha_{2}
\end{array}\right), A_{0}=\left(\begin{array}{cc}
0 & -1 \\
\omega^{2} & 2 \xi \omega
\end{array}\right), A_{1}=\left(\begin{array}{cc}
0 & 0 \\
\alpha_{0} & \alpha_{1}
\end{array}\right) .
$$

The associated characteristic matrix reads as

$$
\begin{aligned}
\mathbb{M}(s) & =s I+s \mathrm{e}^{-\tau s} B-C-E \mathrm{e}^{-\tau s} \\
& =\left(\begin{array}{cc}
s & -1 \\
\omega^{2}-\alpha_{0} \mathrm{e}^{-\tau s} & s-\alpha_{2} s \mathrm{e}^{-\tau s}+2 \omega \xi-\alpha_{1} \mathrm{e}^{-\tau s}
\end{array}\right),
\end{aligned}
$$

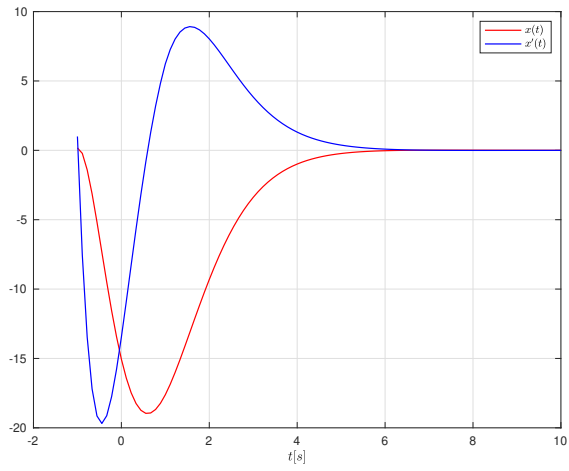

Figure 1. Response of the oscillator 19 subjected to the stabilizing MID property

so that the characteristic quasipolynomial function is $\Delta(s)=\left(-\alpha_{2} s^{2}-\alpha_{1} s-\alpha_{0}\right) \mathrm{e}^{-\tau s}+s^{2}+2 \xi \omega s+\omega^{2}$.

Following Theorem 1, we use the MID stabilizing property by forcing the multiplicity 5 of the real spectral value at:

$$
s_{0}=\left(\sigma \xi^{2}-\sigma-\xi\right) \omega,
$$

where $\sigma(\xi)=\sqrt{-3\left(\xi^{2}-1\right)^{-1}}$ and setting the delay to $\tau=\frac{\sigma}{\omega}$. one computes the appropriate gains:

$$
\begin{aligned}
& \alpha_{0}=-\omega^{2}\left(4 \xi^{3} \sigma-4 \xi \sigma+12 \xi^{2}-13\right) \mathrm{e}^{-(\xi \sigma+3)}, \\
& \alpha_{1}=-2 \omega\left(\sigma \xi^{2}-2 \sigma-\xi\right) \mathrm{e}^{-(\xi \sigma+3)}, \\
& \alpha_{2}=\mathrm{e}^{-(\xi \sigma+3)}
\end{aligned}
$$

Hence, we compute the spectral projection onto the generalized eigenspace $\mathcal{M}_{s_{0}}$ explicitly by the Dumford integral (11), following [14, Section 3.2].

$$
P_{s_{0}} \phi=\operatorname{Res}_{s=s_{0}}\left\{\mathrm{e}^{s} \Delta^{-1}(s) \mathcal{K}\left(s_{0}\right) \phi\right\},
$$

where $\phi={ }^{T}\left(\varphi \varphi^{\prime}\right) \in \mathcal{C}\left([-\tau, 0], \mathbb{C}^{2}\right)$ is the history function, Res is the residue and

$\mathcal{K}\left(s_{0}\right) \phi=D \phi+\int_{-\tau}^{0}\left[s_{0} \mathrm{~d} \mu(\theta)+\mathrm{d} \eta(\theta)\right] \int_{0}^{-\theta} \mathrm{e}^{-s z} \phi(z+\theta) \mathrm{d} z$.

To illustrate the large-time behavior of the trivial solution $x(t)$ of (5), we consider $\xi=\frac{1}{2}, \omega=1$ and the history function $\varphi(\theta)=0.4392434197 \theta^{8}+\theta^{7}-$ $3.648426084 \theta^{3}-3.338574638 \theta^{2}-0.4144356357 \theta+$ 0.05592390768 which satisfies $P_{s_{0}} \phi=0$ as in Theorem 13.

\section{CONCLUSION}

By this paper we extended the multiplicity-induced-dominancy property to the generic secondorder neutral delay equation enabling a stabilizing delayed-feedback design. The proposed design strategy has been employed to exponentially stabilize an 
oscillator. This new extension suggests that investigating this property for more general functional differential equations is an interesting open problem which is of our interest. In particular, a natural question is to generalize the proposed result for a high order equation as well as equations with vectorvalued state. For practical implementation, it is also important to consider robustness aspects of the proposed control design.

\section{REFERENCES}

[1] R. Bellman and K. Cooke. Differential-difference equations. Academic Press, New York, 1963.

[2] I. Boussaada and S-I. Niculescu. Computing the codimension of the singularity at the origin for delay systems: The missing link with Birkhoff incidence matrices. 21st International Symposium on Mathematical Theory of Networks and Systems, pages $1-8,2014$.

[3] I. Boussaada and S-I. Niculescu. Characterizing the codimension of zero singularities for time-delay systems. Acta Applicandae Mathematicae, 145(1):47-88, 2016.

[4] I. Boussaada and S. I. Niculescu. Tracking the algebraic multiplicity of crossing imaginary roots for generic quasipolynomials: A Vandermonde-based approach. IEEE Transactions on Automatic Control, 61:1601-1606, 2016.

[5] I. Boussaada and S-I. Niculescu. On the dominancy of multiple spectral values for time-delay systems with applications. IFAC-PapersOnLine, 51(14):55 - 60, 2018. 14th IFAC Workshop on Time Delay Systems TDS 2018.

[6] I. Boussaada, S-I. Niculescu, A. El Ati, R. Perez-Ramos, and K. Trabelsi. Multiplicity-induced-dominancy in parametric second-order delay differential equations: Analysis and application in control design. (submitted), page 32pp, 2019.

[7] I. Boussaada, S-I. Niculescu, S. Tliba, and T. Vyhlídal. On the coalescence of spectral values and its effect on the stability of time-delay systems: Application to active vibration control. Procedia IUTAM, 22(Supplement C):75 $-82,2017$.

[8] I. Boussaada, S-I. Niculescu, and K. Trabelsi. Toward a decay rate assignment based design for time-delay systems with multiple spectral values. In Proceeding of the 23rd International Symposium on Mathematical Theory of Networks and Systems, pages 864-871, 2018.

[9] I. Boussaada, S. Tliba, S-I. Niculescu, H. Unal, and T. Vyhlídal. Further remarks on the effect of multiple spectral values on the dynamics of time-delay systems. application to the control of a mechanical system. Linear Algebra and its Applications, 542:589 - 604, 2018. Proceedings of the 20th ILAS Conference, Leuven, Belgium 2016.

[10] I. Boussaada, H. Unal, and S-I. Niculescu. Multiplicity and stable varieties of time-delay systems: A missing link. In Proceeding of the 22nd International Symposium on Mathematical Theory of Networks and Systems, pages 16, 2016.

[11] K-L. Cooke and P. van den Driessche. On zeroes of some transcendental equations. Funkcial. Ekvac., 29(1):77-90, 1986.

[12] R.D. Driver, D.W. Sasser, and M.L. Slater. The equation $x^{\prime}(t)=a x(t)+b x(t-\tau)$ with "small" delay. The American Mathematical Monthly, 80(9):990-995, 1973.

[13] M.V.S. Frasson. Large time behaviour for functional differential equations with dominant eigenvalues of arbitrary order. Journal of mathematical analysis and applications, 360(1):278-292, 2009.

[14] M.V.S. Frasson and S.M.V. Lunel. Large time behaviour of linear functional differential equations. Integral Equations and Operator Theory, 47(1):91-121, 2003.
[15] Keqin Gu, Vladimir L. Kharitonov, and Jie Chen. Stability of time-delay systems. Control Engineering. Birkhäuser Boston, Inc., Boston, MA, 2003.

[16] J. K. Hale and S. M. Verduyn Lunel. Introduction to functional differential equations, volume 99 of Applied Mathematics Sciences. Springer Verlag, New York, 1993.

[17] N. D. Hayes. Roots of the transcendental equation associated with a certain difference-differential equation. Journal of the London Mathematical Society, s1-25(3):226-232, 1950.

[18] T. Insperger, J. Milton, and G. Stépán. Acceleration feedback improves balancing against reflex delay. Journal of the Royal Society Interface, 10(79):20120763, 2013.

[19] D. Ma, I. Boussaada, C. Bonnet, S-I. Niculescu, and J. Chen. Multiplicity-induced-dominancy extended to neutral delay equations:towards a systematic pid tuning based on rightmost root assignment. In The 2020 American Control Conference, 2020.

[20] D. Ma and J. Chen. Delay margin of low-order systems achievable by pid controllers. IEEE Transactions on Automatic Control, 64(5):1958-1973, 2018.

[21] Dan Ma, Islam Boussaada, Catherine Bonnet, SilviuIulian Niculescu, and Jie Chen. Multiplicity-InducedDominancy extended to neutral delay equations: Towards a systematic PID tuning based on Rightmost root assignment. In ACC 2020 - American Control Conference, Denver, France, July 2020.

[22] G. Mazanti, I. Boussaada, and S-I. Niculescu. Multiplicity-induced-dominancy for delay-differential equations of retarded type. arXiv preprint arXiv:2002.06128, 2020.

[23] G. Mazanti, I. Boussaada, S-I. Niculescu, and Y. Chitour. Effects of roots of maximal multiplicity on the stability of some classes of delay differential-algebraic systems: The lossless propagation case. arXiv preprint arXiv:2002.00078, 2020.

[24] W. Michiels and S-I. Niculescu. Stability and stabilization of time-delay systems, volume 12 of Advances in Design and Control. SIAM, 2007.

[25] N. Olgac and R. Sipahi. An exact method for the stability analysis of time delayed linear time-invariant (lti) systems. IEEE Transactions on Automatic Control, 47(5):793-797, 2002

[26] J.R. Partington and C. Bonnet. $h_{\infty}$ and BIBO stabilization of delay systems of neutral type. Systems \& Control Letters, 52(3-4):283-288, 2004.

[27] G. Pólya and G. Szegö. Problems and Theorems in Analysis, Vol. I: Series, Integral Calculus, Theory of Functions. Springer-Verlag, New York, Heidelberg, and Berlin, 1972.

[28] G.J. Silva, A. Datta, and S.P. Bhattacharyya. New results on the synthesis of pid controllers. IEEE transactions on automatic control, 47(2):241-252, 2002.

[29] R. Sipahi, S. i. Niculescu, C. T. Abdallah, W. Michiels, and K. Gu. Stability and stabilization of systems with time delay. IEEE Control Systems, 31(1):38-65, Feb 2011.

[30] G. Stépán. Retarded Dynamical Systems: Stability and Characteristic Functions. Pitman research notes in mathematics series. Longman Sci. and Tech., 1989.

[31] K. Walton and J. E. Marshall. Direct method for tds stability analysis. IEE Proceedings D - Control Theory and Applications, 134(2):101-107, 1987. 


\section{Appendix: Proof of Lemma 3}

Let $z_{0}=\sigma+i \omega \in \mathbb{R}^{*}+i \mathbb{R}$ be as in the statement. Thanks to Corollary 1 1 , one may assume that $\sigma>0$. Since $z_{0}$ is a root of $\hat{\Delta}$, one has

$$
\mathrm{e}^{z_{0}}\left(z_{0}^{2}-6 z_{0}+12\right)=z_{0}^{2}+6 z_{0}+12
$$

and therefore, in particular, $\left|\mathrm{e}^{z_{0}}\right|^{2}\left|z_{0}^{2}-6 z_{0}+12\right|^{2}=\left|z_{0}^{2}+6 z_{0}+12\right|^{2}$, which in turn yields

$$
\begin{aligned}
\left(\omega^{4}+\left(2 \sigma^{2}-12 \sigma+12\right) \omega^{2}+\sigma^{4}-12 \sigma^{3}+60 \sigma^{2}-144 \sigma+144\right) \mathrm{e}^{2 \sigma}= & \omega^{4}+\left(2 \sigma^{2}+12 \sigma+12\right) \omega^{2} \\
& +\sigma^{4}+12 \sigma^{3}+60 \sigma^{2}+144 \sigma+144 .
\end{aligned}
$$

Furthermore, since $\mathrm{e}^{2 \sigma}$ is lower bounded by $1+2 \sigma+2 \sigma^{2}+\frac{4}{3} \sigma^{3}$, one deduces that

$$
\begin{aligned}
& \left(2 \sigma+2 \sigma^{2}+\frac{4}{3} \sigma^{3}\right) \omega^{4}+\left(\left(2 \sigma^{2}-12 \sigma+12\right)\left(1+2 \sigma+2 \sigma^{2}+\frac{4}{3} \sigma^{3}\right)-2 \sigma^{2}-12 \sigma-12\right) \omega^{2} \\
& +\left(\sigma^{4}-12 \sigma^{3}+60 \sigma^{2}-144 \sigma+144\right)\left(1+2 \sigma+2 \sigma^{2}+\frac{4}{3} \sigma^{3}\right)-\sigma^{4}-12 \sigma^{3}-60 \sigma^{2}-144 \sigma-144<0 .
\end{aligned}
$$

Now, setting $\Omega=\omega^{2}$, we define $f: \Omega \in \mathbb{R} \rightarrow \mathbb{R}$ the following second degree polynomial

$$
\begin{aligned}
f(\Omega) & =\left(2 x+2 x^{2}+\frac{4}{3} x^{3}\right) \Omega^{2}+\left(\left(2 x^{2}-12 x+12\right)\left(1+2 x+2 x^{2}+\frac{4}{3} x^{3}\right)-2 x^{2}-12 x-12\right) \Omega \\
& +\left(x^{4}-12 x^{3}+60 x^{2}-144 x+144\right)\left(1+2 x+2 x^{2}+\frac{4}{3} x^{3}\right)-x^{4}-12 x^{3}-60 x^{2}-144 x-144,
\end{aligned}
$$

the discriminant of which is given by

$$
D(x)=x^{5} \tilde{D}(x), \quad \text { where } \quad \tilde{D}(x)=-\frac{256}{3} x^{3}+256 x^{2}+320 x+768 .
$$

Since $x>0$, the sign of the discriminant $D$ is equal to that of $\tilde{D}$, which admits a unique real root given by

$$
x_{0}=\frac{(59+8 \sqrt{43})^{\frac{2}{3}}+2(59+8 \sqrt{43})^{\frac{1}{3}}+9}{2(59+8 \sqrt{43})^{\frac{1}{3}}},
$$

owing to the Cardan-Tartaglia method. Hence, the discriminant $D$ admits zero as solution and a unique non-zero real solution $x_{0}$, on the one hand. On the other hand, it is negative in the interval $\left(x_{0},+\infty\right)$ and tends towards $-\infty$ at $\infty$. Consequently, the discriminant $D$ is strictly positive for every $x \in\left(0, x_{0}\right)$.

In what follows, one is only interested in the latter interval in which the discriminant $D$ is strictly positive. In this case, $f$ must admit two real roots, given by

$$
\Omega_{ \pm}(x)=\frac{\left(-2 x^{3}+9 x^{2} \pm 2 \sqrt{-12 x^{4}+36 x^{3}+45 x^{2}+108 x}+3 x\right) x}{2 x^{2}+3 x+3} .
$$

The aim, now, is to determine a bound for the square of the frequency $\Omega$. First, One may remark that the quantity given by

$$
\Omega_{+}(x)-\Omega_{-}(x)=\frac{8 x \sqrt{-3\left(x^{3}-3 x^{2}-\frac{15}{4} x-9\right) x}}{2 x^{2}+3 x+3}
$$

is strictly positive for every $x \in\left(0, x_{0}\right)$, so that $\Omega_{+}$is the greatest solution. Therefore, we shall investigate the maximum of the branch $\Omega_{+}$, by studying the vanishing of its first derivative, i.e.,

$$
\begin{aligned}
\Omega_{+}^{\prime}(x)= & -\frac{6 x\left(4 x^{4}-15 x^{2}-45 x-9\right) \sqrt{-4 x\left(x^{3}-3 x^{2}-\frac{15}{4} x-9\right)}}{\left(-4 x^{4}+12 x^{3}+15 x^{2}+36 x\right)^{\frac{1}{3}}\left(2 x^{2}+3 x+3\right)^{2}} \\
& -\frac{8 \sqrt{3} x\left(6 x^{5}+9 x^{4}-\frac{27 x^{3}}{2}-\frac{297 x^{2}}{4}-108 x-\frac{243}{2}\right)}{\left(-4 x^{4}+12 x^{3}+15 x^{2}+36 x\right)^{\frac{1}{3}}\left(2 x^{2}+3 x+3\right)^{2}}=0 .
\end{aligned}
$$

Or, equivalently, one may investigate the vanishing of its numerator, that is,

$$
\begin{aligned}
-\left(\left(24 x^{4}-90 x^{2}-270 x-54\right) \sqrt{-4 x\left(x^{3}-3 x^{2}-\frac{15}{4} x-9\right)}+8 \sqrt{3}\left(6 x^{5}+9 x^{4}-\frac{27 x^{3}}{2}-\frac{297 x^{2}}{4}\right.\right. & \\
& \left.\left.-108 x-\frac{243}{2}\right)\right) x=0 .
\end{aligned}
$$



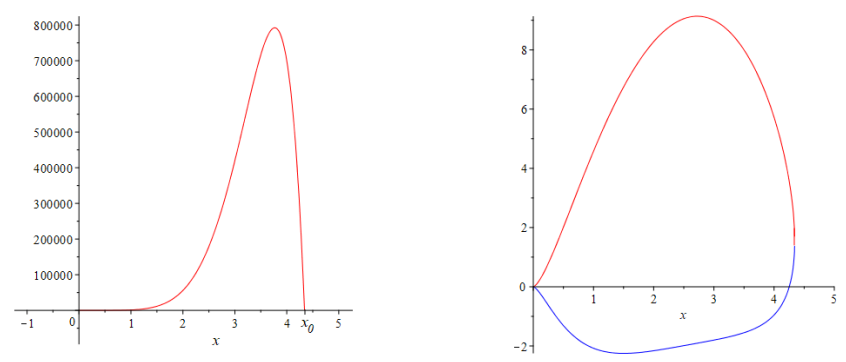

Figure 2. Left: the discriminant $D$ of $f$. Right: graph of $\Omega_{+}$(red) and $\Omega_{-}$(blue).
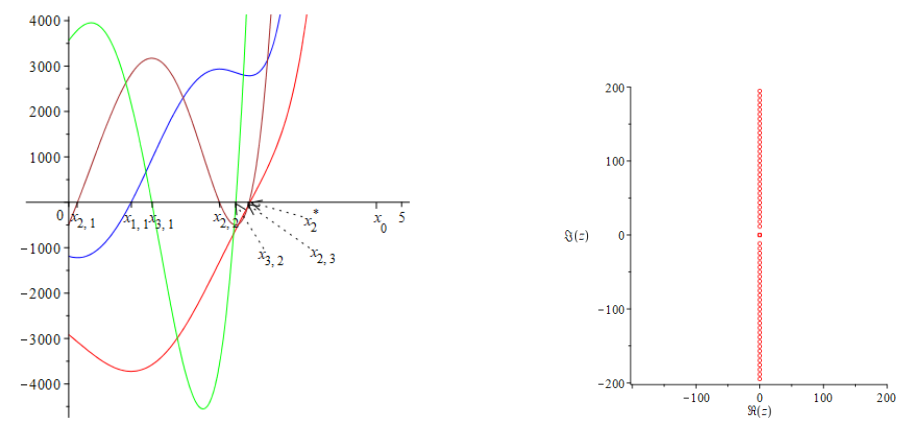

Figure 3. Left: graphs of $P$ (red), $P^{\prime}$ (blue), $P^{2}$ (brown) and $P^{3}$ (green). Right: spectrum distribution of quasipolynomial $\hat{\Delta}$.

By isolating the term $\sqrt{-4 x\left(x^{3}-3 x^{2}-\frac{15}{4} x-9\right)}$

$$
-48(2 x-3)\left(8 x^{7}-36 x^{6}-18 x^{5}+81 x^{4}+594 x^{3}-243 x^{2}-1188 x-2916\right)\left(2 x^{2}+3 x+3\right)^{2}=0 .
$$

The polynomial $2 x-3$ admits one positive root, $x_{1}^{*}=\frac{3}{2}$, corresponding to the point which minimizes the solution $\Omega_{-}$, while the polynomial $2 x^{2}+3 x+3$ is strictly positive. Hence, let us investigate the polynomial

$$
P(x)=8 x^{7}-36 x^{6}-18 x^{5}+81 x^{4}+594 x^{3}-243 x^{2}-1188 x-2916 .
$$

To do so, we need to lower the degree to 4 by computing its third-order derivative

$$
P^{(3)}(x)=1680 x^{4}-4320 x^{3}-1080 x^{2}+1944 x+3564,
$$

the discriminant of which is negative. More precisely, it admits exactly two real roots denoted by $x_{3,1}$ and $x_{3,2}$ and which may be explicitly computed by the Ferrari method. Furthermore, one may remark that $0<x_{3,1}<x_{3,2}<x_{0}$. As a result, the above polynomial has an alternating sign, which means that the second-order derivative of $P$, i.e.,

$$
P^{\prime \prime}(x)=336 x^{5}-1080 x^{4}-360 x^{3}+972 x^{2}+3564 x-486,
$$

has an alternating monotonicity. Namely, it increases from $P^{\prime \prime}(0)<0$ to $P^{\prime \prime}\left(x_{3,1}\right)>0$, it decreases from $P^{\prime \prime}\left(x_{3,1}\right)$ to $P^{\prime \prime}\left(x_{3,2}\right)<0$ and by computing its limit at $\infty$, one may see that it increases again from $P^{\prime \prime}\left(x_{3,2}\right)$ to $\infty$. Then, one deduces that the polynomial given in 22 admits three positive roots denoted by $x_{2,1}, x_{2,2}$ and $x_{2,3}$. Approximating these roots by a numerical algorithm, one infers that $x_{2,1}<x_{3,1}<x_{2,2}<x_{3,2}<$ $x_{2,3}<x_{0}$. Along the same lines, one may deduce that the derivative of $P$,

$$
P^{\prime}(x)=56 x^{6}-216 x^{5}-90 x^{4}+324 x^{3}+1782 x^{2}-486 x-1188,
$$

admits one positive root, denoted by $x_{1,1}$ such that $x_{2,1}<x_{1,1}<x_{3,1}$. Then, with the same analysis, one may also deduce that the polynomial $P$ admits a unique positive root denoted by $x_{2}^{*}$ such that $x_{2,3}<x_{2}^{*}<x_{0}$. Using a numerical algorithm, one may approximate this unique solution by $\left\{x_{2}^{*} \approx 2.72\right\}$, which corresponds to the point that maximizes the solution $\Omega_{+}$at $\Omega_{+}^{*} \approx 9.13$. As a result, $\omega$ is bounded by $\omega^{*} \approx 3.02$, that is, $0<\omega \leq 3.02<\pi$ as required. 